\title{
ABERRANT AXONAL PATHS IN REGENERATED GOLDFISH RETINA AND TECTUM OPTICUM FOLLOWING INTRAOCULAR INJECTION OF OUABAIN
}

\author{
C.A.O. STUERMER ${ }^{1, *}$, A. NIEPENBERG ${ }^{2}$ and H. WOLBURG ${ }^{2}$ \\ 'Max-Planck-Institut für Entwicklungsbiologie, Abteilung Physikalische Biologie, Spemannstrasse, 35; and \\ ${ }^{2}$ Abteilung für submikroskopischè Pathologie und Neuropathologie, Universität Tübingen, D-7400 Tübingen \\ (F.R.G.)
}

(Received April 2nd, 1985; Revised version received May 6th, 1985; Accepted May 7th, 1985)

Key worrds: goldfish retinotectal axon - ouabain-induced degeneration - regeneration - horseradish peroxidase labeling - erratic pathway

Following ouabain-induced degeneration, the neural retina and the retinotectal axons regenerate. The pathways of regenerated retinal ganglion cell axons in retina and in tectum are visualized by labeling with horseradish peroxidase (HRP) applied to the optic nerve. In retina, the axons exhibit highly abnormal courses, including extensive fascicle crossing, hairpin loops and circular routes. In tectum, retinal axon fascicles are not neatly aligned in a normal fascicle fan. Instead, long and short fascicles are mixed, and take erratic routes, crossing each other and crossing the tectal equator.

The regenerative capacity in the goldfish retinotectal system following optic nerve section [1] or pharmacological destruction of the neural retina by ouabain [10] is a striking phenomenon.

With optic nerve section, the retinal ganglion cells, their axons and the intraretinal axon order remains intact and new axonal sprouts are emitted from the cut nerve stumps [9]. On regrowing to their synaptic target sites in the tectum, the regenerating axons make many pathway mistakes $[5,11]$; however, they establish a fiber fascicle array in tectum which largely resembles the normal order [11,13]. In contrast, ouabain injections lead to degeneration of the entire retina, including ganglion cells and their axons, and all intraretinal axonal order is lost. The retina is created de novo probably both from the ouabain-insensitive neuronal precursor cells at the marginal growth zone and from cells in the outer nuclear layer [10]. New axons emerge from the newborn ganglion cells, leave the eye and course towards tectum and make functional connections [8]. However, their pathways within the retina and on the tectum are quite abnormal. In this report, we describe the surprisingly severe pathway aberrations made by ganglion cell axons upon regrowth after ouabain-induced degeneration.

Five microliters of ouabain $\left(10^{-4} \mathrm{M}\right)(6$ fish, both eyes) were injected intraocularly

*Author for correspondence. 
in 1-2-year-old-goldfish (7-10 cm body length). Following survival periods of 15 and 17 days and 3, 4 and 6 months, horseradish peroxidase (HRP) was applied to the optic nerve to label retrogradely axons and their parent ganglion cells in retina and anterogradely axons and their terminal arbors in tectum. Three days later, whole mounts of retina and tectum were prepared and reacted in the chromogens odianisidine [2] and diaminobenzidine (DAB) to visualize the HRP [11, 12]. In order to flat mount the tectum, it was, if necessary, slit from the caudal pole along the tectal equator, the midline between dorsal and ventral hemitecta. Fiber pathways were examined microscopically and recorded in camera lucida tracings or photographed. Retinal and tectal whole mounts of normals and regenerates after nerve cut were available from earlier experimental series [11-13].

General observations. 13 and 17 days after ouabain injection, HRP application to the optic nerve labeled a few, randomly located ganglion cells in the retina and a few lightly stained axons in retina and tectum. This suggests that the majority of retinal axons in the optic nerve and most likely their parent ganglion cells had degenerated [10]. The few ganglion cells and their axons which were labeled, were either the first to regenerate and reach the tectum, or they had survived the ouabain treatment.

Three, 4 and 6 months after ouabain infection, many more ganglion cells were present in scattered distributions (Fig. 2a) but their density was always lower than in normals (approximately $30 \%$ ) or in nerve cut regenerates. Ganglion cells of the 3 major classes were present, i.e. small, medium and large, indicating that there was no particular cell type which was lost completely after ouabain treatment.
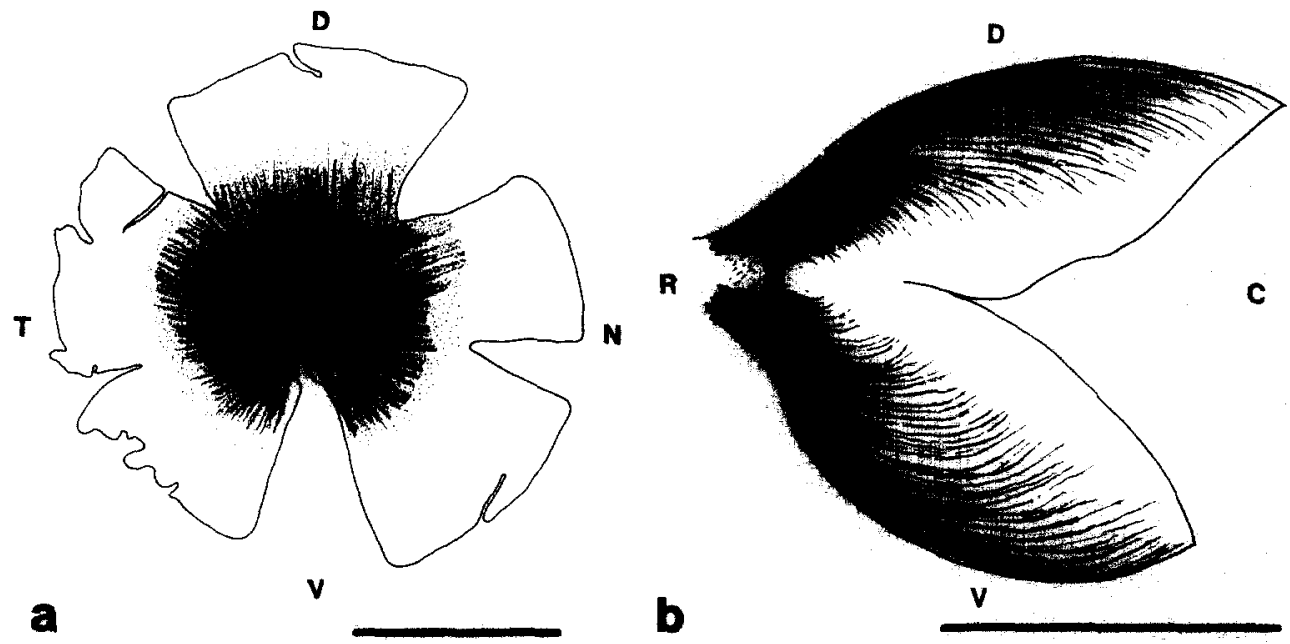

Fig. 1. a: camera lucida tracing of a whole mount of a normal retina after HRP application to the optic nerve, showing HRP-labeled ganglion cells in a central annulus and their axons running radially towards the optic disk. Bar $=2 \mathrm{~mm}$. b: camera lucida tracing of a tectal whole mount showing the anterogradely labeled fiber fascicles in the tectal fiber layer after HRP application to the optic nerve. Note the orderly arrangement of fascicles in two fan-like arrays over the dorsal and ventral hemitectum. $B a r=2 \mathrm{~mm}$. $T$, temporal; N, nasal; V, ventral; D, dorsal; R, rostral; C, caudal; NR, nucleus rotundus. 

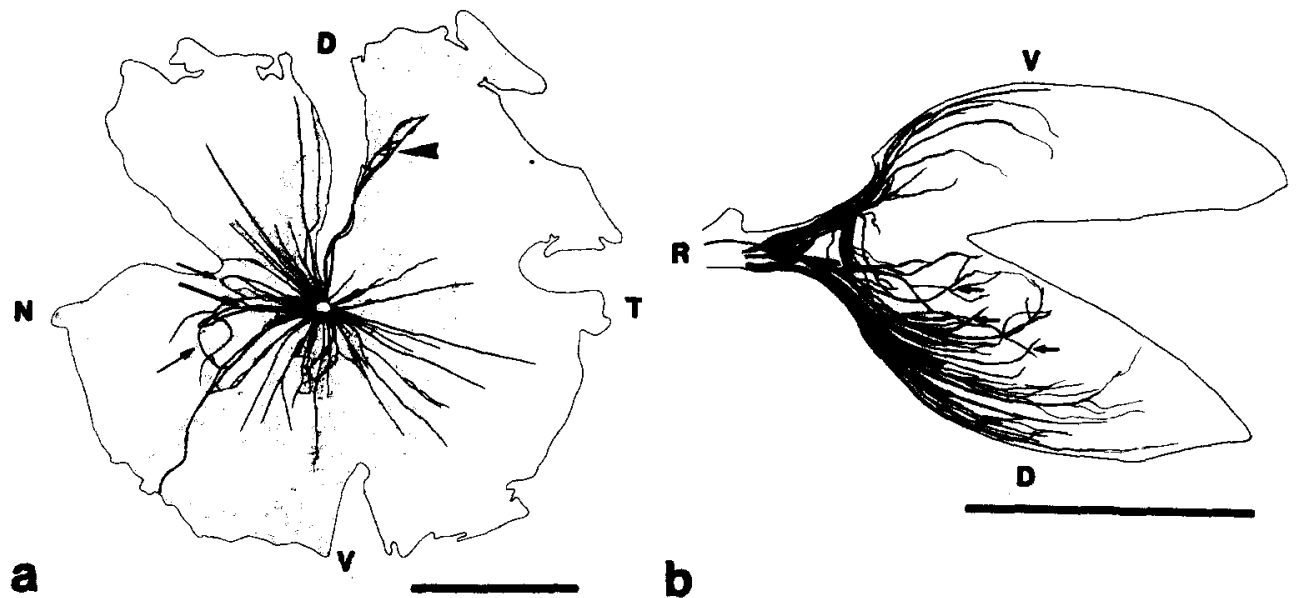

C

b

Fig. 2. Camera lucida tracings of a retina and its contralateral tectum in a 3-month ouabain regenerate, illustrating the abnormal courses of retinal axons, labeled by HRP application to the optic nerve. a: note the abnormal fascicle routes in retina, like circles and hairpin loops (small arrows), fascicle crossing (large arrow) and fiber exchanges between fascicles (arrowhead). b: tectal fascicles lack the fan-like ordering, travel in abnormal paths and cross each other (arrows). Bars in a and b: $2 \mathrm{~mm}$. Abbreviations as in Fig. 1.
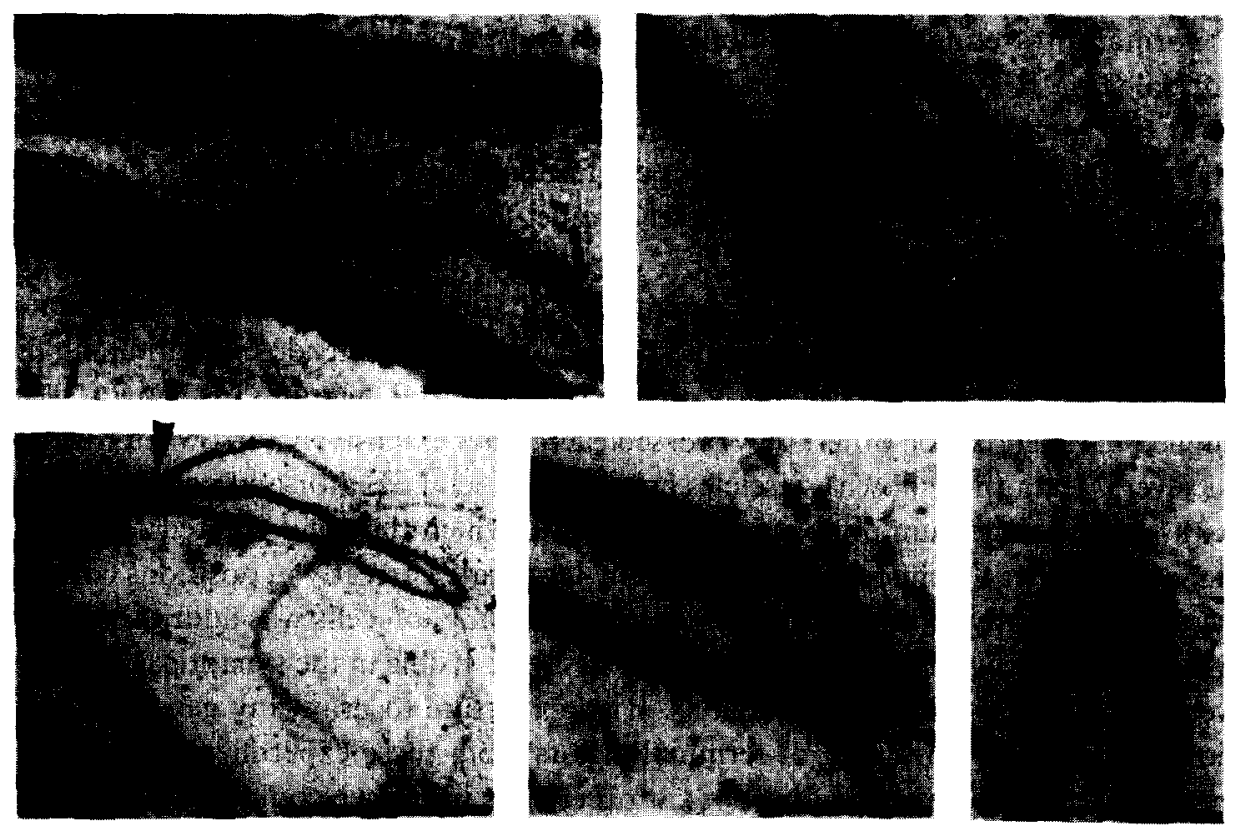

Fig. 3. Photomicrographs of abnormal fiber routes of the retina shown in Fig. 2a. a: arrowhead points to a circular fascicle path. Note a smaller fascicle joining a larger one. $b$ : this figure shows the region marked by an arrowhead in Fig. 2a, and illustrates fiber exchanges between fascicles. c: a hairpin loop and a fascicle crossing over two others (arrowhead). d: hairpin loop of Fig. $2 b$ at higher magnification and two fascicles which merge and join the looping bundle. e: a single fiber (arrowhead) joins a looping fascicle. Bars in a, b and d, $100 \mu \mathrm{m} ; \mathrm{c}, 500 \mu \mathrm{m} ; \mathrm{e}, 25 \mu \mathrm{m}$. 
Axon disorder in retina. While normally most ganglion cell axons emerge from their cell body either at its centrally oriented pole or laterally, in ouabain regenerates a substantial number of axons arose abnormally from the cell body's pole facing the retinal periphery.

In the normal retina (Fig. 1a), axons (here from ganglion cells in a broad annulus) take radially directed routes [3]. Since the retina grows by adding new neurons at the retinal margin [7], axons from younger (more peripheral) generations pass over ganglion cells of previous generations and join fascicles made up of older axons [3].

The axonal pathways in oubain regenerates exhibited severe deviations from the normal order, as exemplified by one typical retina of a 4-month regenerate shown in Fig. 2a. As readily appreciable from Fig. 2a, the ouabain regenerate contained fewer but thicker axon fascicles being formed from the axons of ganglion cells located at widely separated positions. Moreover, fascicles and single axons crossed one another frequently (long arrow in Fig. 2a). The most striking aberrations were hairpin loops or circular courses (Figs. 2a and 3a, c-e). In several instances, ganglion cells in the vicinity of these abnormally routed fascicles contributed their axons to the errant bundle and were thus trapped in the fascicle's circular path.

Such drastic misroutings are never seen in normal retinae or nerve-cut regenerates and are unique to ouabain regenerates.

Disorderly path of tectal fascicles. Tecta innervated by retinal axons with highly abnormal intraretinal routes exhibited abnormal fascicular paths in tectum. In the normal (Fig. 1b), and similarly in the nerve-cut regenerate, fascicles of retinal axons form two fan-like arrays from their rostroperipheral point of entry over the dorsal and ventral hemitectum [11-13] with a continuum from short to long fascicles [12]. Most fascicles take curved paths, travel parallel to each other and end at the tectal equator $[11,12]$.

The fascicle path of ouabain regenerates (Fig. 2b) lacked a consistent alignment of short rostrocentral and long peripherocaudal fascicles. Instead, short and long fascicles were intermixed, particularly in the rostrocentral tectum. Instead of being aligned parallel to each other, they crossed each other extensively (arrows in Fig. 2b). In several tecta (not shown), fascicles crossed the tectal equator and continued their path in the hemitectum opposite to the one into which they had entered. The questions of whether the misrouted axons finally terminate correctly in positions correlating retinotopically to the position of their parent ganglion cells was addressed in two fish. Only a few lightly stained terminal arbors were visible in the synaptic layer. They were in retinotopically correct regions. It is premature to draw any general conclusions on correct positioning of terminal arbors from these experiments, since there might be many more terminal arbors at other sites which we could not yet identify. The analysis of retinotopic ordering is in progress.

The foregoing report illustrates highly abnormal pathways of ganglion cell axons in retina and tectum following ouabain-induced retinal degeneration. Aberrant paths are not only found in the tissue directly affected by the poisoning agent but also in the target tissue of retinal axons, the tectum, which has remained untouched. Thus, this system is well suited for investigating the effects on axonal behavior and naviga- 
tion in the target region which perturbation of retinal axonal order might produce.

During the continuous retinal growth [7], the development of orderly fiber routes in retina is created by new axons following older ones in vitreal positions [3] resulting from a higher affinity of growth cones for axons of older ganglion cells than for growth cones of their own generation [3]. In ouabain regenerates, the order of ganglion cell birth is most likely very different from the normal [10]. The existence of large fascicles composed of numerous axons of different retinal regions and the merger of fascicles could be interpreted either as a result of growth cones liking to follow other axons, or as a result of mutual affinities between growth cones. The affinity of axons to others is substantiated by the observation that individual axons join fascicles, even those in errant paths or vice versa. Despite their errant paths, the axons ultimately adopt routes directed towards the optic disk. These radial routes and particularly the changes of axonal courses into centripetal direction may be indicative for the possible existence - and, if so, maintenance after ouabain intoxication - of directional cues along which axons can become oriented [6].

In the normal, the spatiotemporal pattern of retinal growth [7] bears direct consequences on the fiber order in tectum in which fascicles are ordered by age, i.e. by their origin from ganglion cells in annular retinal regions [12].

In ouabain regenerates, the fascicle arrangements in the tectal fiber layer appears erratic, perhaps as a consequence of abnormal developmental events in retinal reconstitution. The fascicle disorder in ouabain regenerates is even more compelling when compared to that of the nerve-cut regenerate $[11,12]$. The latter contains numerous pathway mistakes; however, the gross order of fiber fascicles regained on the tectum is reminiscent of the normal order [13]. We thus speculate that the reorganization of fiber fascicle order in tectum may be highly dependent on the maintenance of the spatiotemporal order of their parent ganglion cells in retina. With the absence of normal order of cells in retina, the conventional fiber paths may not be restored.

Our current approach of labeling regenerating axons in the optic nerve restrains our considerations to those ganglion cell axons which have left the eye and we inevitably miss possibly existing axons which stray and wander within the retina without finding their exit [6]. Such axonal paths and the early stages of axonal outgrowth and behavior before leaving the eye has to be established in further experiments with different methods [6].

In further experiments we will determine the spatiotemporal sequence of ganglion cell formation and axon outgrowth in ouabain regenerates, in order to test its influence on the formation of abnormal fiber paths. We will also be investigating whether or not retinotopy is reestablished in the tectum $[1,5]$.

We are grateful to $\mathbf{R}$. Groemke-Lutz for her skillful photographical reproductions. We thank E. Kalko and T. Gutensohn for their excellent technical assistance and Drs. F. Bonhoeffer and S. Chang for corrections and K. Ralinofsky for typing the manuscript. 
1 Attardi, D.G. and Sperry, R.W., Preferential selection of central pathways by regenerating optic fibers, Exp. Neurol., 7 (1963) 46-64.

2 Coleman, D.R., Scalia, F. and Cabrales, E., Light and electron microscopic observations on the anterograde transport of horseradish peroxidase in the optic pathway in the mouse and rat, Brain Res., 102 (1976) $156-163$.

3 Easter, S.S., Bratton, B. and Scherer, S.S., Growth-related order of the retinal fiber layer in goldfish, J. Neurosci., 4 (1984) 2173-2190.

4 Easter, S.S., Jr. and Stuermer, C.A.O., An evaluation of the hypothesis of shifting terminals in goldfish optic tectum, J. Neurosci., 4 (1984) 1052-1063.

5 Egert, U., Kalko, E. and Stuermer, C.A.O., Retinotopic termination and abnormal pathways of regenerating optic axons in the goldfish tectum, Soc. Neurosci. Abstr., 10 (1984) 143.

6 Halfter, W. and Deiss, S., Axon growth in embryonic chick and quail retinal whole mounts in vitro, Dev. Biol., 102 (1984) 344-355.

7 Johns, P.R. and Easter, S.S., Growth of the adult goldfish eye. Increase in retinal cell number, J. Comp. Neurol., 176 (1977) 331-342.

8 Kästner, R. and Wolburg, H., Functional regeneration of the visual system in teleosts. Comparative investigations after optic nerve crush and damage of the retina, Z. Naturforsch., 37 (1982) 1274-1280.

9 Lanners, N.H. and Grafstein, B., Early stages of axonal regeneration in the goldfish optic tract: an electron microscopic study, J. Neurocytol., 9 (1980) 733-751.

10 Maier, W. and Wolburg, H., Regeneration of the goldfish retina after exposure to different doses of ouabain, Cell Tiss. Res., 202 (1979) 99-118.

11 Stuermer, C.A.O. and Easter, S.S., Jr., A comparison of the normal and regenerated retinotectal pathways of goldfish, J. Comp. Neurol., 223 (1984) 57-76.

12 Stuermer, C.A.O. and Easter, S.S., Jr., Rules of order in the retinotectal fascicles of goldfish, J. Neurosci., 4 (1984) 1045-1051.

13 Stuermer, C.A.O., Restoration of growth-related order of regenerated optic axon fascicles in goldfish, Soc. Neurosci. Abstr., 10 (1984) 465. 